\title{
The generating identity of Cauchy-Schwarz-Bunyakovsky inequality
}

\begin{abstract}
Antonino M. Sommariva
Antonino M. Sommariva received a "Laurea in Ingegneria Elettronica" degree from the University of Palermo in 1977. Currently, he is a full professor in the "Dipartimento di Elettronica per l'Automazione" at the University of Brescia (Italy). His main research interests are perturbation methods, and the fundamental problems of classical circuit theory.
\end{abstract}

\section{Introduction}

The Cauchy-Schwarz-Bunyakovsky inequality (CSB-inequality for short) is well-known to pure and applied mathematicians for its application in different areas of mathematics (algebra, analysis, geometry, probability theory, etc.). A complete account of its both intricate and intriguing history can be found in a well documented paper by Schreiber [3] or in the delightful book by Steele [4].

This important relationship can be formulated as follows:

CSB-inequality - Let $V$ be a real or complex inner product vector space, and let $x, y$ be elements of $V$. Then

$$
|x \cdot y| \leqslant\|x\||| y||
$$

where equality holds if and only if $x$ and $y$ are linearly dependent.

Was Sie immer schon über die Schwarzsche Ungleichung wissen wollten, sich aber nie zu fragen getrauten: Wo bleibt eigentlich die Differenz? Die Beweise in den Lehrbüchern der linearen Algebra oder der Funktionalanalysis sind auf Knappheit getrimmt und geben darüber keine Auskunft. Es gibt allerdings eine Lagrange zugeschriebene Identität, die diese Differenz als Quadrat eines gewissen schiefsymmetrischen Ausdrucks darstellt. Der Autor der vorliegenden Arbeit ist der Sache auf den Grund gegangen und findet im Rahmen des Tensorprodukts $V \otimes V$ eine einleuchtende und allgemeingültige Erklärung. 
To this author's best knowledge, identities giving rise to the CSB-inequality have been obtained in the past, but for particular cases only. Thus, Cauchy derived his version of the inequality by deleting some summands in an arithmetical identity ascribed to Lagrange, i.e.

$$
\begin{gathered}
\left(a \alpha+a^{\prime} \alpha^{\prime}+a^{\prime \prime} \alpha^{\prime \prime}+\ldots\right)^{2}+\left(a \alpha^{\prime}-a^{\prime} \alpha\right)^{2}+\left(a \alpha^{\prime \prime}-a^{\prime \prime} \alpha\right)^{2}+\left(a^{\prime} \alpha^{\prime \prime}-a^{\prime \prime} \alpha^{\prime}\right)^{2}+\ldots \\
=\left(a^{2}+a^{\prime 2}+a^{\prime \prime 2}+\ldots\right)^{2}\left(\alpha^{2}+\alpha^{\prime 2}+\alpha^{\prime \prime 2}+\ldots\right)^{2}
\end{gathered}
$$

where $a, a^{\prime}, a^{\prime \prime}, \ldots, \alpha, \alpha^{\prime}, \alpha^{\prime \prime}, \ldots$ are two sequences of $n$ real numbers each. The same identity appears (adopting the self-evident notation of [2, pp. 33, 62]) in exterior algebra or the theory of spatial vectors as

$$
(\boldsymbol{a} \cdot \boldsymbol{b})^{2}+|\boldsymbol{a} \wedge \boldsymbol{b}|^{2}=\boldsymbol{a}^{2} \boldsymbol{b}^{2} \quad \text { or } \quad(\boldsymbol{a} \cdot \boldsymbol{b})^{2}+|\boldsymbol{a} \times \boldsymbol{b}|^{2}=\boldsymbol{a}^{2} \boldsymbol{b}^{2}
$$

respectively.

In the context of functional analysis, Courant and Hilbert [1, p. 49] reported (without reference) an identity which can be rewritten as

$$
(f, g)^{2}+\frac{1}{2} \iint(f(x) g(\xi)-f(\xi) g(x))^{2} \mathrm{~d} x \mathrm{~d} \xi=(f, f)(g, g)
$$

where $f$ and $g$ are real-valued functions which are piecewise continuous in some finite interval $G \subset \mathbb{R}$.

This paper proposes an identity which gives rise to the CSB-inequality for the general case, based on the concept of tensor product of vector spaces. That is, first, a fundamental relationship is established between the inner product in a vector space $V$ and a particular inner product in the tensor product of $V$ by itself. Then, a Pythagorean relation, involving symmetric and antisymmetric parts of a tensor, is derived and manipulated to obtain the desired identity. Finally, two examples are presented.

\section{The identity behind the CSB-inequality}

Let $V$ be a real or complex inner product vector space, and let $V \otimes V$ be the tensor product of $V$ by itself. If $\left\{h_{m}\right\}$ is any basis of $V$, then $\left\{h_{m} \otimes h_{n}\right\}$ is a basis of $V \otimes V$, and it is referred to as the basis induced by $\left\{h_{m}\right\}$. An inner product can be introduced also in $V \otimes V$ by specifying proper values for the inner products of the induced basis elements. In particular, if the basis $\left\{h_{m}\right\}$ is orthonormal, i.e.,

$$
h_{p} \cdot h_{q}=\delta_{p q},
$$

and the basis $\left\{h_{m} \otimes h_{n}\right\}$ is assumed as orthonormal, i.e.,

$$
\left(h_{p} \otimes h_{q}\right) \cdot\left(h_{r} \otimes h_{s}\right)=\delta_{p r} \delta_{q s},
$$

then it is easily seen that for any $a, b, c, d \in V$ the following formula holds:

$$
(a \otimes b) \cdot(c \otimes d)=(a \cdot c)(b \cdot d) .
$$

In fact, having set

$$
a=\sum a_{p} h_{p}, \quad b=\sum b_{q} h_{q}, \quad c=\sum c_{r} h_{r}, \quad d=\sum d_{s} h_{s},
$$


it turns out that

$$
\begin{aligned}
(a \otimes b) \cdot(c \otimes d) & =\left(\sum a_{p} h_{p} \otimes \sum b_{q} h_{q}\right) \cdot\left(\sum c_{r} h_{r} \otimes \sum d_{s} h_{s}\right) \\
& =\sum a_{p} b_{q}\left(h_{p} \otimes h_{q}\right) \cdot \sum c_{r} d_{s}\left(h_{r} \otimes h_{s}\right) \\
& =\sum a_{p} b_{q} \bar{c}_{r} \bar{d}_{s}\left(h_{p} \otimes h_{q}\right) \cdot\left(h_{r} \otimes h_{s}\right) \\
& =\sum a_{p} b_{q} \bar{c}_{r} \bar{d}_{s} \delta_{p r} \delta_{q s}=\sum a_{p} b_{q} \bar{c}_{p} \bar{d}_{q}=\sum a_{p} \bar{c}_{p} \sum b_{q} \bar{d}_{q} \\
& =(a \cdot c)(b \cdot d) .
\end{aligned}
$$

Under these assumptions, the symmetric and the antisymmetric part of the tensor product $x \otimes y$, denoted $\operatorname{Sym}(x \otimes y)$ and $\operatorname{Asym}(x \otimes y)$ respectively, are orthogonal:

$$
\begin{aligned}
& \operatorname{Sym}(x \otimes y) \cdot \operatorname{Asym}(x \otimes y)=\frac{1}{2}(x \otimes y+y \otimes x) \cdot \frac{1}{2}(x \otimes y-y \otimes x) \\
& =\frac{1}{4}\left(\|x\|^{2}\|y\|^{2}-\|y\|^{2}\|x\|^{2}-(x \cdot y)(y \cdot x)+(y \cdot x)(x \cdot y)\right)=0
\end{aligned}
$$

and thus the Pythagorean relationship holds:

$$
\|\operatorname{Sym}(x \otimes y)\|^{2}+\|\operatorname{Asym}(x \otimes y)\|^{2}=\|x \otimes y\|^{2} .
$$

Moreover, one can obtain

$$
\|x \otimes y\|^{2}=(x \otimes y) \cdot(x \otimes y)=\|x\|^{2}\|y\|^{2}
$$

and

$$
\begin{aligned}
\|\operatorname{Sym}(x \otimes y)\|^{2} & =\frac{1}{2}(x \otimes y+y \otimes x) \cdot \frac{1}{2}(x \otimes y+y \otimes x) \\
& =\frac{1}{4}\left(\|x\|^{2}\|y\|^{2}+\|y\|^{2}\|x\|^{2}+(x \cdot y)(y \cdot x)+(y \cdot x)(x \cdot y)\right) \\
& =\frac{1}{2}\left(\|x\|^{2}\|y\|^{2}+|x \cdot y|^{2}\right) .
\end{aligned}
$$

Hence, substitution of (2) and (3) into (1) yields the wanted identity:

$$
|x \cdot y|^{2}+2\|\operatorname{Asym}(x \otimes y)\|^{2}=\|x\|^{2}\|y\|^{2} .
$$

According to (4), the term which completes the (squared) CSB-inequality can be interpreted and calculated as twice the squared norm of the antisymmetric part of the tensor product of the involved elements, provided that the basis induced by an orthonormal basis of $V$ is assumed as an orthonormal basis of $V \otimes V$.

Sometimes, $V \otimes V$ has some given inner product, and it is desirable to use it. In this case, one must verify that the induced basis $\left\{h_{m} \otimes h_{n}\right\}$ is orthonormal with respect to this inner product. 


\section{Examples}

In this section, two examples are considered which extend the results (Lagrange's identity, Courant-Hilbert's identity) mentioned in the introduction. In both of them, the tensor product $V \otimes V$ has a given usual inner product, and the basis of $V \otimes V$ induced by an orthonormal basis of $V$ is orthonormal with respect to this inner product.

Let $\mathbb{C}^{M \times N}$ be the space of $M \times N$ matrices over $\mathbb{C}$, and let $\mathbb{C}^{M \times N}$ be endowed with its usual inner product, i.e., if $\boldsymbol{A}, \boldsymbol{B} \in \mathbb{C}^{M \times N}$,

$$
\boldsymbol{A} \cdot \boldsymbol{B}:=\operatorname{trace}(\tilde{\boldsymbol{A}} \overline{\boldsymbol{B}})
$$

where the tilde stands for transpose. For any $\boldsymbol{A}, \boldsymbol{B} \in \mathbb{C}^{M \times N}$, consider the column partitions

$$
\boldsymbol{A}=\left[\begin{array}{llll}
\boldsymbol{a}_{1} & \boldsymbol{a}_{2} & \cdots & \boldsymbol{a}_{N}
\end{array}\right], \quad \boldsymbol{B}=\left[\begin{array}{llll}
\boldsymbol{b}_{1} & \boldsymbol{b}_{2} & \cdots & \boldsymbol{b}_{N}
\end{array}\right],
$$

then set

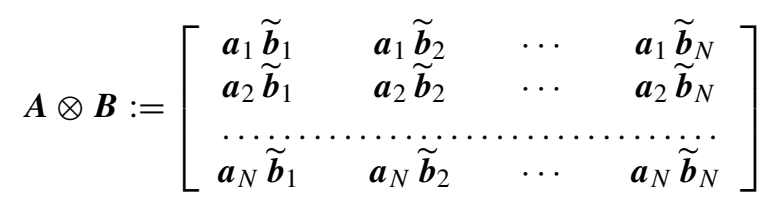

and observe that $\mathbb{C}^{M \times N} \otimes \mathbb{C}^{M \times N}=\mathbb{C}^{(M N) \times(M N)}$. Therefore, according to (4), if $\boldsymbol{A}=$ $\left(a_{p q}\right)$ and $\boldsymbol{B}=\left(b_{r s}\right)$, the term which completes the (squared) CSB-inequality in this example amounts to

$$
\frac{1}{2} \sum_{p, q, r, s}\left|a_{p q} b_{r s}-b_{p q} a_{r s}\right|^{2} .
$$

The real case of the above formula with $N=1$ corresponds to Lagrange's identity.

Let $C_{\mathrm{pw}}(G, \mathbb{C})$ be the space of complex-valued functions which are piecewise continuous in some finite interval $G \subset \mathbb{R}^{N}$, and let $C_{\mathrm{pw}}(G, \mathbb{C})$ be endowed with its usual inner product, i.e. if $f, g \in C_{\mathrm{pw}}(G, \mathbb{C})$

$$
f \cdot g:=\int \cdots \int f\left(x_{1}, \ldots, x_{N}\right) \bar{g}\left(x_{1}, \ldots, x_{N}\right) \mathrm{d} x_{1} \cdots \mathrm{d} x_{N} .
$$

For any $f, g \in C_{\mathrm{pw}}(G, \mathbb{C})$, set

$$
f \otimes g: G^{2} \rightarrow \mathbb{C}
$$

$$
(f \otimes g)\left(x_{1}, \ldots, x_{N}, \xi_{1}, \ldots, \xi_{N}\right):=f\left(x_{1}, \ldots, x_{N}\right) g\left(\xi_{1}, \ldots, \xi_{N}\right)
$$

and observe that $C_{\mathrm{pw}}(G, \mathbb{C}) \otimes C_{\mathrm{pw}}(G, \mathbb{C})=C_{\mathrm{pw}}\left(G^{2}, \mathbb{C}\right)$. Therefore, according to (4), the term which completes the (squared) CSB-inequality in this example amounts to

$$
\frac{1}{2} \int \cdots \int\left|f\left(x_{1}, \ldots, x_{N}\right) g\left(\xi_{1}, \ldots, \xi_{N}\right)-g\left(x_{1}, \ldots, x_{N}\right) f\left(\xi_{1}, \ldots, \xi_{N}\right)\right|^{2} \mathrm{~d} x_{1} \cdots \mathrm{d} \xi_{N} .
$$

The real case of the above formula with $N=1$ corresponds to Courant-Hilbert's identity. 


\section{References}

[1] Courant, R.; Hilbert, D.: Methods of Mathematical Physics I. Wiley-Interscience, 1989.

[2] Gröbner, W.: Matrizenrechnung. Bibliographisches Institut, Mannheim 1966.

[3] Schreiber, P.: The Cauchy-Bunyakovsky-Schwarz Inequality. In: Hermann Graßmann - Werk und Wirkung. Ernst-Moritz-Arndt-Universität, Greifswald 1995, 64-70.

[4] Steele, J.M.: The Cauchy-Schwarz Master Class: An Introduction to the Art of Mathematical Inequalities. MAA Problem Book Series, Cambridge 2004.

Antonino M. Sommariva

Università degli Studi di Brescia

Via Branze, 38

I-25123 Brescia, Italy

e-mail: sommariv@ing.unibs.it 